Fisher's Exact Test were used to compare questionnaire scores and symptom prevalence between those who were breastfed and attended nursery and those who were not. The longitudinal analysis of the LBBS was performed using multi-level mixed effects models.

Results At four, ten and sixteen months of age infants who were breastfed had a lower symptom scores and a smaller prevalence of a variety of respiratory symptoms than those who were not. Some of these findings showed a dose-response relationship. This relationship was no longer present at twenty-two months of age. Longitudinally, breastfeeding was shown to reduce symptom scores over the first twenty-two months of life.

At four months infants who attended nursery had a higher prevalence of snoring and were more likely to attend the GP with respiratory symptoms. At ten, sixteen and twenty-two months of life nursery attenders had higher symptom scores and a higher prevalence of various respiratory symptoms than non-attendees. Longitudinally over time, nursery attendance increased symptom scores over the first twenty-two months of life. Nursery attendance reduced the quality of life of both the infant and their families due to respiratory symptoms at ten and sixteen months of age.

Conclusion Breastfeeding was protective of respiratory symptoms over the first 22 months of life. Contrastingly, nursery attendance increased respiratory symptoms and reduced the quality of life of both infants and their family over the first 22 months of life.

\section{G448 JUVENILE DERMATOMYOSITIS ASSOCIATED WITH INTERSTITIAL LUNG DISEASE: WOULD ANTI-MDA5 ANTIBODY DETECTION IMPROVE EARLY DIAGNOSIS AND MANAGEMENT?}

K Gallagher, P Bale, K Armon. Paediatric Rheumatology, Addenbrooke's Hospital, Cambridge, UK

\subsection{6/archdischild-2018-rcpch.437}

Aims We describe a case of anti-MDA5 antibody positive Juvenile Dermatomyositis(JDM) associated with interstitial lung disease(ILD) and propose early antibody detection may guide management and improve outcome.

Methods A 15-year-old male presented with a 3 month history of pain and stiffness in his right ankle, left knee and hand joints associated with mild weakness. A blistering rash on his extremities developed into classical JDM facial rash with periorbital swelling, Gottron papules and paranychial ulcers. Initial investigations included: EMG study showing myopathy; MRI buttock and skin biopsy both consistent with dermatomyositis (see table 1). He started hydroxychloroquine, weekly methotrexate injections and high dose prednisolone including fortnightly 3 day intravenous pulses.

Results Within one month, he developed a knee staphylococcus aureus arthritis which required prolonged antibiotics. Despite minimal respiratory symptoms, his lung function test showed a restrictive pattern with reduced diffusion(FVC-7.57 predicted, TLC-4.18, DLC -2.2). ILD with patchy consolidation was confirmed on imaging. IV immunoglobulins(IVIG) were added. Two weeks later, he developed chest pain and shortness of breath, with clinical signs of surgical emphysema confirmed radiologically, with apical pneumothoraces, pneumomediastinum and pneumopericardium. The air leaks were secondary to bronchial perforations secondary to JDM related ILD. Although the rash, arthritis and myositis responded to treatment, his ILD progressed. He required admission to PICU for respiratory support and was covered for infection including pneumocystis pneumonia. Further IVIG and IV methylprednisolone was given and mycophenolate commenced. However, his deterioration necessitated ventilation and transfer for ECMO. His bloods showed anti-MDA5 positive antibodies. Plasmapheresis, cyclophosphamide and rituximab were given but despite best efforts, he died from ILD secondary to JDM.

\begin{tabular}{|c|c|c|}
\hline & Diagnosis & $\begin{array}{l}\text { PICU } \\
\text { admission }\end{array}$ \\
\hline $\mathrm{Hb}(\mathrm{g} / \mathrm{L})$ & 116 & 103 \\
\hline WCC(10*9/L) & 6.9 & 9.2 \\
\hline Neut(10*9/L) & 3.4 & 7.34 \\
\hline Platelets(10*/L) & 211 & 256 \\
\hline ALT(U/L) & 131 & 122 \\
\hline GGT(U/L) & 85 & - \\
\hline $\operatorname{ALP}(\mathrm{U} / \mathrm{L})$ & - & 60 \\
\hline $\mathrm{ESR}(\mathrm{mm})$ & 2 & 88 \\
\hline ANCA & Negative & - \\
\hline ANA & Negative & - \\
\hline $\begin{array}{l}\text { Myositis- } \\
\text { antibodies }\end{array}$ & Negative & \\
\hline CK(IU/L) & 164 & 462 \\
\hline Ferritin(ug/L) & - & 2367 \\
\hline LD(U/L) & - & 74 \\
\hline
\end{tabular}

Conclusion Anti-MDA5 antibodies have been reported in rapidly progressive ILD associated with JDM. Despite the usual manifestations responding to treatment, his lung disease was unremittingly progressive. The clinical features associated with this rare form included the unusual vasculitic lesions periungually and on the palms/soles. At diagnosis, we recommend requesting full myositis-specific antibodies including antiMDA5 which may help guide management. The advent of aggressive staphylococcus auerus arthritis delayed the use of additional immunosuppressants once ILD was diagnosed and IVIG was used whilst treating the infection. Early aggressive immunosuppression is recommended.

\section{G449 LUNG FUNCTION AT FOLLOW-UP OF VERY PREMATURELY BORN YOUNG PEOPLE - IMPACT OF BRONCHOPULMONARY DYSPLASIA}

${ }^{1} \mathrm{~S}$ Morris, ${ }^{1} \mathrm{C}$ Harris, ${ }^{1} \mathrm{~A}$ Lunt, ${ }^{2} \mathrm{~J}$ Peacock, 'A Greenough. 'Women and Children's Health, School of Life Course Sciences, Faculty of Life Sciences and Medicine, King's College London, London, UK; ${ }^{2}$ Primary care and Public Health Sciences, King's College London, London, UK

\subsection{6/archdischild-2018-rcpch.438}

Aims Bronchopulmonary dysplasia (BPD) is a common adverse outcome of very premature birth. Studies have given conflicting results as to whether BPD is associated with long-term lung function impairment, but few have included populations exposed to routine use of antenatal steroids and postnatal surfactant. To determine, according to BPD status, the lung function of 16 to 18 year old, very prematurely born young 